\title{
Better in-hospital outcome among hypertensive subjects developing acute myocardial infarction-study in a tertiary cardiac care center in Bangladesh
}

\author{
Prabir Kumar Das ${ }^{1}$, Sayed Md Hasan², Salehuddin Siddique ${ }^{3}$, Munzur Murshed ${ }^{4}$, A K M Fazlur Rahman 5
}

\begin{abstract}
Background:In-hospital complications and mortality in hypertensives developing acute myocardial infarction(AMI) may be different from those of normotensive counterpart. The aim of the current study was to analyze in-hospital complication and outcome of AMI in hypertensive patients and compare it with age and sex matched normotensive AMI patients. Methods: In-hospital complications of $\mathbf{1 1 2}$ hypertensive patients with AMI admitted over a period of 1 year (April 2014 to March 2015) were compared with the control group. Location and types of AMI were determined by ECG. Patients were considered to be hypertensive if they were taking antihypertensive treatment or were found to have a systolic blood pressure (SBP) e"140 $\mathrm{mmHg}$ and/or a diastolic blood pressure (DBP) e" $90 \mathrm{mmHg}$ on repeated measurements. Both groups were studied prospectively. The results were analyzed by SPSS software. Results:Out of total 112 patients 69 were male and $\mathbf{4 3}$ were female in each group. Male: female ratio was 1.6:1. Mean age of the study population was $67.3 \pm 10.2$ yrs (range 41 to $83 \mathrm{yrs}$ ). Mean blood pressure values were $145.7 \pm 11.5 / 88.3 \pm 8.9 \mathrm{mmHg}$ in the hypertensive and
\end{abstract}

$127.3 \pm 9.7 / 75.8 \pm 5.6 \mathrm{mmHg}$ in normotensive group respectively. A significantly higher prevalence of diabetes, dyslipidemia, chronic kidney disease, stroke and peripheral vascular disease were found in the hypertensive compared with the normotensive subjects. Hypertensive AMI patients had higher left ventricular ejection fraction compared with the normotensives $(0.51 \pm 0.13$ vs $0.47 \pm 0.15)$. A higher frequency of paroxysmal atrial fibrillation(AF) $(\mathbf{1 0 . 7 \%}$ vs $7.1 \%, \mathrm{P}<0.05)$ and a lower frequency of atrioventricular block (5.4\% vs $8.0 \%)$, ventricular fibrillation ( $2.7 \%$ vs $4.5 \%)$, cardiogenic shock $(4.5 \%$ vs $9.8 \%, \mathrm{P}<0.01)$ and a lower inhospital mortality $(6.2 \%$ vs $10.7 \%, P<0.01)$ were found among the hypertensives compared with the normotensives. Conclusion: Hypertensive AMI patients had a significantly higher incidence of AF, lower incidence of cardiogenic shock and an overall better inhospital outcome compared with the normotensives, probably owing to a better preserved left ventricular function, prior use of cardioprotective drugs and yet undefined mechanism.

Key Words: Hypertension, AMI, Outcome

(Bangladesh Heart Journal 2016; 31(1) : 10-17)

Introduction:

Hypertension is a major risk factor for the development of AMI. It is considered to participate in the pathogenesis of atheromatous plaque, its ulceration and thrombosis ${ }^{1}$.

1. Associate Professor, Dept.of Cardiology, Cox's Bazar Medical College.

2. Senior Consultant(Card), Cox's Bazar Sader Hospital.

3. Assistant Professor(Card), Chittagong Medical College, Chittagong.

4. Associate Professor (Card), Chittagong Medical College, Chittagong.

5. Professor of Interventional Cardiology, Bangabandhu Sheikh Mujib Medical University, Dhaka
Prevalence of hypertension in AMI varies from $31 \%$ to $50 \%$ in various studies ${ }^{2}$. There is a paucity of data on hemodynamic and electrical complications of AMI in hypertensive patients. Mauri et $\mathrm{al}^{3}$ reported an increased incidence of sudden death in AMI patients with a history of hypertension. On the other hand Abrignani et $\mathrm{al}^{4}$ found lower incidence of shock, ventricular fibrillation, conduction disturbances, cardiac rupture and intracardiac thrombus in hypertensive patients with first MI, while atrial fibrillation was more common in these patients. More recently Rombek et $\mathrm{al}^{5}$ found higher incidence of cardiogenic 
shock, pulmonary oedema, ventricular tachycardia and/ or ventricular fibrillation and third degree

atrioventricular(AV) block. Other studies did not show relevant difference for in-hospital and 6 month's mortality in hypertensive and normotensive patients with MI. ${ }^{6}$

Prevalence of hypertension as well as coronary artery disease is increasing in countries of Indian subcontinent, including Bangladesh. Because of increasing number of hypertensive subjects developing acute myocardial infarction, it is an important subject to give attention. Hence, the study was done to estimate the in-hospital complications and outcome of AMI among hypertensive subjects.

\section{Aim of Study}

To analyze the in-hospital complication and outcome of AMI in hypertensive subjects and compare it with age and sex matched normotensive AMI patients.

\section{Material \& Method}

Study design-prospective observational;case control

Inclusion criteria-first onset AMI patient with antecedent hypertension or newly diagnosed hypertension

Exclusion criteria- i.patients with previous history of myocardial infarction.ii.patients with previous baseline ECG findings of left bundle branch block, pre-excitation syndrome

The study was conducted in the coronary care unit of Chittagong Medical College Hospital.The study period was one year (April, 2014 to March, 2015).We found a total of 112 patients in each group.As control, we randomly selected age and sex matched normotensive individuals with AMI. The study protocol was approved by the ethical committee on human research of the institute. Patient's characteristics, location and types of AMI, diagnostic and therapeutic measures undertaken and various complications were recorded. AMI was diagnosed if at least two of the following criteria were met $^{7}$ : (i) typical chest pain lasting for at least $30 \mathrm{~min}$ and not relieved by nitrates (ii) ST segment and/or T-wave changes suggestive of myocardial infarction and (iii) serum troponin- i or creatine phosphokinase $(\mathrm{CK})$ concentration of more then twice the upper limit of normal range. The patents were categorized as having antecedent hypertension if the diagnosis was known by the patients or his attendant to having been made by their family physician, pre-admission records showed that they were hypertension and/or they were receiving anti-hypertensive drugs. A diagnosis of new hypertension were made when BP was $<140 / 90 \mathrm{mmHg}$ on receiving the patient that persisted in that range after the patient's condition settled (in 1-2 hrs). Blood for fasting glucose and lipid profile was drawn within $24 \mathrm{hrs}$ of hospital admission. An individual was considered to be diabetic if he or she was receiving insulin or oral hypoglycemic agents or had symptoms of diabetes with fasting blood glucose $>126 \mathrm{mg} / \mathrm{dl}^{8}$. Dyslipidemia was defined when the patients had history of taking lipid lowering drugs or when any of the lipid fractions was abnormal, for example, serum cholesterol $>160 \mathrm{mg} / \mathrm{dl}$ or $\mathrm{HDL}<35 \mathrm{mg} / \mathrm{dl}$, LDL $>100 \mathrm{mg} /$ dl or triglyceride $>150 \mathrm{mg} / \mathrm{dl}^{8}$. A family history was considered positive when symptomatic coronary artery disease occurred in siblings, parent's sibling's or grand parents before age 45 in male and 55 in female. Blood sample were send for estimation of serum troponin-I, CK-MB (when re-infarction suspected). The patients were treated with medications according to current recomendations. Those presenting with $\mathrm{AMI}$ and a very high blood pressure, a controlled reduction of BP without compromising coronary flow was undertaken with antihypertensive drugs to a range of goal BP of 160-170 $\mathrm{mmHg}$ systolic and $100-110 \mathrm{mmHg}$ diastolic. Thrombolytic therapy with i.v streptokinase was administered to the eligible patients presenting within 12 hours of onset of chest pain. Those presenting with very high $\mathrm{BP}$, therapy was withheld until BP was reduced to less than $160 / 110 \mathrm{mmHg}$ with medication. Intravenous nitroglycerine (dose 0.5 to $5.0 \mathrm{microgm} / \mathrm{min}$ ) was used in patients with hypertensive emergencies, and hypertensives with AMI with or without left ventricular failure. Oral beta-blocker and angiotensin converting enzyme inhibitors were used in all hypertensive AMI patients unless contraindicated. These drugs were also used in all normotensive patients with anterior wall infarction unless contraindicated. Presence of heart failure was assessed clinically according to Killip classification as follows: Class - no evidence of heart failure Class - bi-basilar rales \pm S3, Class III- pulmonary edema (with respiratory distress, diaphoresis, cold extremity), Class IV- cardiogenic shock. Hemodynamic monitoring with central venous and arterial line were done in patients with cardiogenic shock. Patients with acute left ventricular failure was managed with i.v morphine and diuretics. Those developing cardiogenic shock were managed conservatively with i.v.inotropes(dopamine and dobutamine).Arrhythmic complications were managed with appropriate anti-arrhythmic medications, e.g digitalis for atrial fibrillation, i.v lignocaine,amiodarone etc. for ventricular tachycardia, ventricular fibrillation, along with cardioversion in appropriate cases. Temporary pacemaker implantation was done for patients with AV block and hemodynamic instability, followed by implantation of permanent pacemaker in cases of 
anterior wall infarction. Doppler echocardiographic study was done in all patients to assess LV function and mechanical complications post MI.

3.4.Statistical analysis-results were presented as mean \pm standard deviation for continuous variables and numbers (\%) for categorical variables. Student's ' $t$ ' test and chi-square tests were used to determine the level of significance. $P$ value $<0.05$ was considered statistically significant

\section{Results}

A total of 224 subjects were analysed with 112 subjects in each group, There were 69 male(61.6\%) and $43(38.4 \%)$ female in both groups. Male:female ratio was 1.6:1. Mean age of the study population was $67.3 \pm 10.2 \mathrm{yrs}$ ( range 41 to 83 years).Baseline characteristics of the patient population are shown in table I.

Concomitant risk factors of Coronary artery disease (CAD) in the two study groups are shown in table II.

DM, dyslipidaemia and a positive family history of CAD were significantly higher in hypertensives subjects compared with normotensives. Normotensive subjects had a higher prevalence of smoking compared with hypertensives (not statistically significant).

Hypertensive AMI subjects showed a higher prevalence of co-morbidities as shown in table III.
Various diagnostic and therapeutic procedures undertaken in the two study groups are shown in table IV

Anterior wall MI was the commonest in both the study groups followed by inferior MI with no significant difference between them $(47 \%$ vs $46 \%$ and $35 \%$ vs $37 \%$ respectively). Seven percent of hypertensives and 10\% of normotensive subjects had a combined (ant.\& inf.) MI. A significantly higher percentage of hypertensive subjects had NSTEMI compared with the normotensives $(19.9 \%$ vs $15.9 \%, P<0.05)$.

Majority of the hypertensive subjects (69\%) presented to the CCU within 12 hrs of onset of their chest pain. Whereas, $55 \%$ of the normotensive subjects presented within 12 hrs of onset of their chest pain $(p<0.05)$. Thus a greater number of hypertensive subjects were being thrombolysed ( $57.1 \%$ vs $50.9 \%$ ) compared with normotensives, even though patients with BP e"160/ $110 \mathrm{mmHg}$ were excluded from therapy.

Pharmacologic treatment undertaken in the two groups are shown in table $\mathrm{V}$.

A higher percentage of hypertensive subjects received low molecular weight heparin (LMWH) compared with the normotensives consistent with the higher NSTEMI cases among the hypertensives, all of whom received LMWH. Other cardioprotective drugs (beta blockers, ACEI) were used significantly more in hypertensive subjects.

Table-I

Characteristics of hypertensive subjects $(n=112)$

\begin{tabular}{lcc}
\hline Characteristics & Frequency & Percentage (\%) \\
\hline Sex & & \\
$\quad$ Male & 69 & 61.6 \\
$\quad$ Female & 43 & 38.4 \\
Antecedent hypertensionm & 103 & 92.0 \\
$\quad$ Poorly controlled & 38 & 33.9 \\
$\quad$ Well controlled & 65 & 58.0 \\
Anti-hypertensives used : & & \\
Beta blockers + calcium channel blockers & 33 & 29.5 \\
ACE inhibitors / angiotensin receptor blockers & 27 & 24.1 \\
Beta blockers & 13 & 11.6 \\
Angiotensin receptor blockers +Thiazides & 10 & 8.9 \\
Calcium channel blockers & 9 & 8.0 \\
Thiazides & 6 & 5.4 \\
Angiotensin receptor blockers + calcium channel blockers & 3 & 2.7 \\
Other drugs & 2 & 1.8 \\
Newly diagnosed hypertension & 9 & 8.0 \\
\hline
\end{tabular}

*Mean duration of raised BP: $13 \pm 7.4$ yrs 
Table-II

$C A D$ risk factors in the two groups

\begin{tabular}{lccc}
\hline Risk factors & Hypertensive $(\mathrm{n}=112)$ & Normotensive $(\mathrm{n}=112)$ & P value \\
\hline Smoking & $58(51.8)$ & $59(52.7)$ & $\mathrm{NS}$ \\
Diabetes mellitus & $47(42.0)$ & $38(33.9)$ & $<0.05$ \\
Dyslipidemia & $43(38.4)$ & $40(35.7)$ & $<0.05$ \\
Positive F/H of CAD & $29(25.9)$ & $19(17.0)$ & $<0.01$ \\
Obesity & $7(6.2)$ & $6(5.4)$ & $\mathrm{NS}$ \\
\hline
\end{tabular}

Figures within parentheses indicate percentages

Table-III

Co-morbidities in the two groups

\begin{tabular}{lccc}
\hline Co-morbidities & Hypertensive $(\mathrm{n}=112)$ & Normotensive $(\mathrm{n}=112)$ & $\mathrm{P}$ value \\
\hline Chronic obstructive pulmonary disease & $13(11.6)$ & $9(8.0)$ & $<0.01$ \\
Cerebrovascular disease & $11(9.8)$ & $5(4.5)$ & $<0.05$ \\
History of revascularization & $8(7.1)$ & $5(4.5)$ & $\mathrm{NS}$ \\
Chronic kidney disease & $7(6.2)$ & $3(2.7)$ & $<0.05$ \\
\hline
\end{tabular}

Figures within parentheses indicate percentages

Table-IV

Diagnostic and therapeutic procedures $(n=224)$

\begin{tabular}{lccc}
\hline Procedures & Hypertensive $(\mathrm{n}=112)$ & Normotensive $(\mathrm{n}=112)$ & P value \\
\hline Doppler echocardiography & $112(100.0)$ & $112(100.0)$ & - \\
Chest radiography & $27(24.1)$ & $19(17.0)$ & $<0.01$ \\
Hemodynamic monitoring & $12(10.7)$ & $9(8.0)$ & $\mathrm{NS}$ \\
Cardio-pulmonary resuscitation & $8(7.1)$ & $11(9.8)$ & $\mathrm{NS}$ \\
Cardioversion & $7(6.2)$ & $8(7.1)$ & $\mathrm{NS}$ \\
Transvenous pacing & $6(5.4)$ & $5(4.5)$ & $\mathrm{NS}$ \\
\hline
\end{tabular}

Figures within parentheses indicate percentages

Table-V

Drug administered in CCU in two groups

\begin{tabular}{|c|c|c|c|}
\hline Drugs & Hypertensive(n = 112) & Normotensive $(\mathrm{n}=112)$ & $\mathrm{P}$ value \\
\hline Clopidogrel & $97(86.6)$ & $96(85.7)$ & NS \\
\hline LMW heparin & $70(62.5)$ & $63(56.2)$ & NS \\
\hline Thrombolytic (streptokinase) & $64(57.1)$ & $57(50.9)$ & $<0.05$ \\
\hline Oral GTN & $62(55.4)$ & $48(42.9)$ & $<0.01$ \\
\hline ACE inhibitors / angiotensin receptor blockers & $47(42.0)$ & $39(34.8)$ & $<0.05$ \\
\hline Beta blockers & $37(33.0)$ & $26(23.2)$ & $<0.01$ \\
\hline Diuretics (frusemide) & $32(28.6)$ & $24(21.4)$ & $<0.05$ \\
\hline Lignocaine & $25(22.3)$ & $31(27.7)$ & NS \\
\hline GTN i.v. & $23(20.5)$ & $17(15.2)$ & $<0.01$ \\
\hline Dobutamine / Dopamine & $20(17.9)$ & $22(19.6)$ & NS \\
\hline Digoxin & $9(8.0)$ & $7(6.2)$ & NS \\
\hline Calcium channel blockers & $8(7.1)$ & $3(2.7)$ & NS \\
\hline
\end{tabular}

Figures within parentheses indicate percentages 


\section{Evolving Complications:}

Analysis of arrhythmic complications developing in the two study populations shows that a significantly higher prevalence of paroxysmal atrial fibrillation and a lower prevalence of conduction disturbance (AV block) and ventricular arrhythmia were found in hypertensives compared with normotensives Table VII shows the various mechanical complications developing in the two study populations.

Hypertensive AMI paients had a higher prevalence of LV failure (Killip class 2-4).A significantly higher percentage of cardiogenic shock $(4.5 \%$ vs $9.8 \%, p<0.01)$ was found among the normotensive subjects compared with hypertensives. Analysis of ischemic complications (Table VIII) shows that a significantly higher reinfarction rate was present in the hypertensive group.

Mean hospital stay of the two groups were not significantly different $(9 \pm 2.7$ days in hypertensives vs. $9 \pm 1.8$ days in normotensives). Echocardiographic examination revealed a higher left ventricular ejection fraction(LVEF) among the hypertensives compared with the normotensives $(0.51 \pm 0.13$ vs.0.47 \pm 0.15$)$. In-hospital mortality in the two groups are shown in table IX.

There was significantly lower in-hospital mortality among the hypertensive AMI subjects compared with the normotensive AMI subjects.

Table-VI

Arrhythmic complications in the two groups $(n=224)$

\begin{tabular}{lccc}
\hline Arrhythmic Complications & Hypertensive $(\mathrm{n}=112)$ & Normotensive $(\mathrm{n}=112)$ & $\mathrm{P}$ value \\
\hline Sinus tachycardia & $75(67.0)$ & $85(75.9)$ & $<0.01$ \\
Paroxysmal AF & $12(10.7)$ & $8(7.1)$ & $<0.05$ \\
AV block & $6(5.4)$ & $9(8.0)$ & $\mathrm{NS}$ \\
Ventricular fibrillation & $3(2.7)$ & $5(4.5)$ & $\mathrm{NS}$ \\
Ventricular tachycardia & $2(1.8)$ & $3(2.7)$ & $\mathrm{NS}$ \\
\hline
\end{tabular}

Figures within parentheses indicate percentages

Table-VII

Mechanical complications in the two groups

\begin{tabular}{lccc}
\hline Mechanical Complications & Hypertensive $(\mathrm{n}=112)$ & Normotensive $(\mathrm{n}=112)$ & $\mathrm{P}$ value \\
\hline LV failure & $22(19.6)$ & $20(17.9)$ & $\mathrm{NS}$ \\
Cardiogenic shock & $5(4.5)$ & $11(9.8)$ & $<0.01$ \\
Mitral regurgitation & $3(2.7)$ & $2(1.8)$ & $\mathrm{NS}$ \\
LV aneurysm & $3(2.7)$ & $5(4.5)$ & $\mathrm{NS}$ \\
Ventricular septal rupture(VSR) & $2(1.8)$ & $1(0.9)$ & $\mathrm{NS}$ \\
\hline
\end{tabular}

Figures within parentheses indicate percentages

Table-VIII

Ischemic complications in the two groups

\begin{tabular}{lccc}
\hline Ischemic Complications & Hypertensive $(\mathrm{n}=112)$ & Normotensive $(\mathrm{n}=112)$ & $\mathrm{P}$ value \\
\hline Re-infarction & $11(9.8)$ & $7(6.2)$ & $<0.05$ \\
Post Ml angina & $8(7.1)$ & $6(5.4)$ & $\mathrm{NS}$ \\
Infarct expansion & $3(2.7)$ & $2(1.8)$ & $\mathrm{NS}$ \\
\hline
\end{tabular}

Figures within parentheses indicate percentages

Table-IX

In-hospital mortality in the two groups

\begin{tabular}{cccc}
\hline In-hospital mortality & Hypertensive $(\mathrm{n}=112)$ & Normotensive $(\mathrm{n}=112)$ & $\mathrm{P}$ value \\
\hline $7(6.2)$ & $12(10.7)$ & $<0.01$ & \\
\hline
\end{tabular}

Figures within parentheses indicate percentages 


\section{Discussion:}

The role of arterial hypertension in relation to in-hospital outcome in AMI has not been definitely established. Available evidence does not clearly show an increased rate of adverse outcome after AMI including stroke, heart failure and cardiovascular death among hypertensives ${ }^{9}$. Hypertension has been found to be a weak predictor of death in AMI patients by several investigators ${ }^{10}$. Muri et $\mathrm{al}^{3}$ described that the increased risk of hemodynamic complications and death is related to the extend and expansion of $\mathrm{MI}$, an anterior wall involvement, an increased extent of CAD, the presence of severe ventricular arrhythmias and myocardial rupture. Mortality from CAD rises continuously with increasing levels of systolic and diastolic $\mathrm{BP}^{14}$. However the relationship between the two is complex.

Prevalence of hypertension increases with increase in age, which is itself an important predictor of poor outcome in $\mathrm{AMI}^{15}$. Despite a global reduction in mortality from AMI in general population it remains still high among elderly ${ }^{16}$. Mortality from AMI is also high among females ${ }^{4}$. In an attempt to exclude age and sex as confounding factor we matched normotensives AMI patients by these factors. Hypertension was found to be combined with increased associated co-morbidities in the form of cerebrovascular disease, peripheral arterial disease, renal failure, diabetes, dyslipidemia and chronic obstructive airway disease $^{4}$ and on the basis of observations of other authors, greater target organ damage, such as nephropathy, microalbuminuria etc. ${ }^{11}$. Our hypertensive AMI patients had a higher prevalence of co-morbidities (table-2) such as DM, dyslipidemia, chronic kidney disease, chronic obstructive airway disease and history of revascularization which is in agreement with the findings of Ascenzu et al ${ }^{17}$. We did not found any significant difference regarding anterior or inferior location of AMI in our patients but a significantly higher rates of non STelevation $\mathrm{Ml}(19.9 \%$ vs $15.9 \%, \mathrm{P}<0.05)$ was found in hypertensives subjects compared with normotensives. A higher prevalence of non-ST elevated MI(NSTEMI) among hypertensives were also described in epidemiologic studies, where chronic hypertension was found as the most prevalent risk factors ${ }^{13}$. This higher prevalence of NSTEMI cases among the hypertensives may have also contributed to the lower in-hospital complications and mortality which is also in agreement with the findings of Abrigrnin et al ${ }^{4}$. Hypertensive subjects are known to have severe coronary atherosclerosis ${ }^{19}$ which allows development of more collateral circulation. It explains the more NSTEMI cases among the hypertensives.
Most of the studies regarding links between hypertension and $\mathrm{MI}$ consider patients with a previous history of hypertension but data are lacking regarding patients who do not have antecedent hypertension but showed elevated BP values during their hospital stay for AMI. A study on patients with AMI admitted within 6 hours of onset of pain $31.7 \%$ patients had an elevated BP (e"180/ $100 \mathrm{mmHg}$ ) on presentation, only $6.3 \%$ of them had elevated BP after 6 hours though not treated with antihypertensive drugs ${ }^{20}$. We found such new cases of hypertension in $8 \%$ of our patients. We undertook a controlled reduction of BP in our AMI patients with hypertension in the CCU. Patients with long standing uncontrolled hypertension can not tolerate rapid great reduction of $\mathrm{BP}$. The hypertensive myocardium is sensitive to BP lowering below a critical level. A rapid reduction of diastolic BP may potentially be more dangerous to the heart. Result of a recent analysis of AMI treated early with ACE inhibitors suggest caution in patients with antecedent hypertension but with low or normal BP at presentation, as these patients showed a higher first day mortality 25 . The recommended BP target in hypertensive AMI subjects is not completely clear. Further studies are needed to define the issue more clearly.

Analysis of arrhythmic complications showed that only paroxysmal AF was found to be more frequent in our hypertensive patients. Other arrhythmias and AV block were more in normotensive subjects. This is in contrast to the general concept that hypertensive individuals are more prone to cardiac arrhythmias ${ }^{21}$. Abrignani et al ${ }^{4}$ found an almost similar findings in their hypertensive patients with first time MI. A smaller necrotic area and presence of well developed collateral may better preserve conduction pathway, explaining the lesser prevalence of conduction disturbance and ventricular arrhythmias in hypertensive subjects. A higher prevalence of paroxysmal AF among our hypertensive subjects may be related to left atrial enlargement and chronically elevated left atrial pressure 22 . Prevalence of cardiogenic shock was significantly lower in our hypertensive patients $(4.5 \%$ vs $9.8 \%, \mathrm{P}<0.01)$ compared with the normotensives. Cardiogenic shock remains the leading cause of death in patients hospitalized with $\mathrm{AMI}^{18}$ but its incidence has been greatly reduced by the use of reperfusion therapy through mechanical revascularization. Because of lack of mechanical revascularization and IABP facilities we could only undertake pharmacologic reperfusion with i.v. thrombolytic and conservative management of cardiogenic shock with inotropes. A higher percentage of our hypertensive ST elevated MI(STEMI) patients received 
thrombolytic therapy because of their early presentation to the CCU. Among the hypertensive STEMI subjects $69 \%$ presented to CCU within $12 \mathrm{hrs}$. Whereas only $55 \%$ of the normotensive subjects presented before $12 \mathrm{hrs}$. Higher rates of thrombolytic administration to the hypertensive subjects had possibly limited their infarct size as evident by the lower serum enzyme levels and a higher LV ejection fraction on echocardiography.

The lower incidence of cardiogenic shock and a better preserved LV function was responsible for significantly lower in-hospital mortality in the hypertensive AMI patients. Hypertensive subjects had a lower in-hospital mortality compared with the normotensives $(6.2 \%$ vs. $10.7 \%, P<0.01)$. This is probably due to a better preserved LV function with a higher LVEF among the hypertensives $(0.51 \pm 0.13$ vs. $0.47 \pm 0.15)$ compared with the normotensives. This is in accordance with the findings of Abrignani et al ${ }^{4}$ who are the first to show a significantly lower in-hospital complications and morbidity in hypertensive subjects with first time AMI compared with normotensives. This lower in-hospital mortality is in contrast to the findings of Richards et $\mathrm{al}^{23}$ who found the same as $8.1 \%$ in their hypertensive patients compared with $4.4 \%$ among the normotensives. Their hypertensive patients also had a higher incidence of acute LV failure (33\% vs. $24 \%$ ) compared with the normotensives. Literature published so far shows that this is one of the few studies that showed a significantly better in-hospital outcome in hypertensive AMI subjects. Although this better outcome may not be maintained long term because of the greater severity of CAD in hypertensives and higher co-morbidities among them ${ }^{25}$ and an adverse long term outcome is not unusual. Further studies may clarify the issue.

The study has got some important limitations:

i.Besides age and sex other patient characteristics were not excluded as confounding variables. ii.24 hours blood pressure recording of the study population were not possible. iii. primary $\mathrm{PCl}$ was not possible due to lack of facility. iv.only in-hospital outcome data are described here, post discharge follow up and long term complications and mortality data are lacking.

\section{Conclusion:}

Hypertensive AMI subjects had a better in-hospital outcome compared with normotensive AMI patients. These better outcomes is probably related to a better preserved LV function due to less infarct extension in hypertensive and more cases of NSTEMI among them. Early presentation of STEMI in the hypertensive group with higher rates of thrombolytic administration, more use of cardioprotective antihypertensive drugs, presence of extended collaterals in the hypertensives and yet unidentified factors may have also contributed to the better in-hospital outcome.

\section{References:}

1. Libby P. Current concept of pathogenesis of acute coronary syndrome. Circulation 2001;104:365-372

2. Herlitz I, Karlson BW, Richter A,Polak JF,Kronmal RA,Monnilio TA, et al. Prognosis in hypertensive with acute myocardial infarction. J Hypertens 1992;10:265-72

3. Muri F, Maggioni AP, Franzosi MG. A simple electrocardiographic predictor of the outcome of patients with acute myocardial infarction treated with a thrombolytic agents. A GISSI-2 derived analysis. J Am Coll Cardiol 1994;24:600-607

4. Abrignani MG,Dominguez LJ,Biondo G. In-hospital complications of acute myocardial infarction in hypertensive subjects. Am J Hypertens 2005; 18(2):165-170

5. Rombek M, Goch A, Goch T. The clinical course of acute ST-elevation myocardial infarction in patients with hypertension. Kardiologia Polska 2010; 68(2):157-163

6. Majahalme SK,Smith DE, Cooper JV,Bennett DK.Comparison of patients with acute coronary syndrome with and without systemic hypertension. Am J Cardiol 2003;92(3):258-263

7. Nomenclature and criteria for diagnosis of ischemic heart disease. Report of the Joint International Society and Federation of Cardiology/ World Health Organization Task Force on Standardization of Clinical Nomenclature. Circulation 1979;59:607-9

8. Enas EA, Senthilkumar A. Coronary artery disease in Asian Indians: an update and review. Internet $\mathrm{J}$ Cardiol 2002;1(2):10-24

9. Thune JJ,Signororitch J,Kober L,Bombelli M,Hedberg P,Ohrvik J,et al.Effcet of antecedent hypertension and followup blood pressure on outcome after high risk myocardial infarction. Hypertension 2008;51(1):48-54

10. Herlitz J, Karlson BW, Lindqrist J, Sjolin M,Shorman $\mathrm{JE}$, Hare JL,et al.Important predictors for the 10 - 
year mortality rate in patients with acute chest pain or other symptoms consistent with AMI with particular emphasis on the influence of age. Am Heart J 2001;42:624-632

11. Vandenburg M, Hoogen P, Fesckens E, Kerke $\mathrm{N}$, Thomas S, Davis JE, et al. The relationship between blood pressure and mortality due to coronary heart disease among men in different parts of the world. N Engl J Med 2000;342:1-10

12. Leek L, Woodlief LH, Topol EJ. for the GUSTO-1 Investigators: Predictors of 30-day mortality in the era of reperfusion for AMI. Results from an international trial of 41021 patients. Circulation 1995;91:1659-1668

13. Devlin W, Crangg D, Jacks M, Willams RR, Friedman GD,Smith RG, et al. Comparison of outcome in patients with AMI aged $>75$ years with that in younger patients. Am J Cardiol 1995;75:573-576

14. Kannel WB. Blood pressure as a cardiovascular risk factor: Prevention and treatment. JAMA 1996;275:1571-6

15. Ascenzu FD, Gonella A,Anadri G, Leano R. Comparison of mortality rates in women versus men presenting with ST-segment elevation MI. Am J Cardiol 2011;107(5):651-654

16. Hardai $D$, Behar $S$, Wallentin L,Longberg M,Lawrent S. A prospective survey of the characteristics, treatment and outcome of patients with acute coronary syndrome in Europe and the Mediterranean basin: the Euro Heart Survey of acute coronary syndrome(Euro Heart Survey ACS). Eur Heart J 2002;23(15):1190-1201

17. Chobanian AV, Bakris GL, Black HR. National Heart Lung and Blood Institute Joint National Committee on Prevention, Detection, Evaluation and Treatment of High Blood Pressure, National High Blood Pressure Education Program Coordinating Committee: The Seventh Report of the Joint National Committee on Prevention, Detection,
Evaluation and Treatment of High Blood Pressure. JAMA 2003;289:2500-2572

18. Gibson T.C. Blood pressure levels in acute myocardial infarction. Am Heart J 1978;96(4): 475-480

19. Polese A, Decesare N, Montoris P,Nicolson G,Gross $\mathrm{A}$, Palmer $\mathrm{BF}$,et al. Upward shift of the lower range of coronary flow regulation in hypertensive patients with hypertrophy of the left ventricle. Circulation 1991;83:845-53

20. Avanzini F, Ferrario G, Santoro L,Grosso A. Risk and benefit of early treatment of acute myocardial infarction with an angiotensin converting enzyme inhibitor in patients with a history of arterial hypertension: analysis of the GISSI-3 database. Am Heart J 2002;144:1018-1025

21. Novo S, Barbagello M, Abrignani MG,Eisenhofer G,Kaplan MM. Increased prevalence of cardiac arrhythmia and transient episodes of myocardial ischemia in hypertensive individuals with left ventricular hypertrophy but without clinical history of coronary artery disease. Am J Hypertens 1997; 10:843-851

22. Barbier P, Aliotto G, Guazzi M,Sung J. Left atrial function and ventricular filling in hypertensive patients with paroxysmal AF. J Am Coll Cardiol 1994;24:165-170

23. Valente S,Lazzeri C,Vecchiopol S,Levy D,Manacia $\mathrm{G}$,Salmon M,et al. Predictors of in-hospital mortality after $\mathrm{PCl}$ for cardiogenic shock. Int J Cardiol 2007;114(2):176-182

24. Mark A, Richard A, Gary J, Nicholi S, Richard WT. Antecedent hypertension and heart failure after myocardial infarction. J Am Coll Cadiol 2002;39(2):1182-118

25. Gustafson F, Korber L, Torp L, Pederson C,Hunt SC,Barlow GK,et al. Long term prognosis after acute myocardial infarction in patients with a history of arterial hypertension. TRACE study group. Eur Heart J 1998;19:588-594 